\title{
A DEVELOPMENTAL STUDY OF GOAL AND ENTRANCE GRADIENTS IN A UNILINEAR MAZE IN HUMAN SUBJECTS
}

\author{
TERUKO TAKEMOTO,* TATSURO YATABE,** SHINKURO IWAHARA, \\ AND MASAKO OGAWA
}

\author{
Nara Women's University* \& Kyoto University**
}

Haslerud (1) studied the form and interaction of goal and entrance gradients in a unilinear maze composed of four entrance units, four free units, and four goal units in this order. Using rats as subjects, he found, besides other main results, that turning responses in the free units were greatly affected by rewarded turning responses in the goal units as compared with those in the entrance units.

The purpose of this research was to investigate whether similar results would be obtained in human subjects with two mazes structurally identical to Haslerud's maze and how such results, whatever they might be, would be a function of age.

\section{EXPERIMENT I}

Subjects. Five groups of different ages were used. They are shown below :

\begin{tabular}{rccc}
\hline Age & Male & Female & Total \\
\hline 4 & 11 & 11 & 22 \\
5 & 17 & 13 & 30 \\
6 & 14 & 14 & 23 \\
8 & 10 & 10 & 20 \\
10 & 10 & 10 & 20 \\
\hline
\end{tabular}

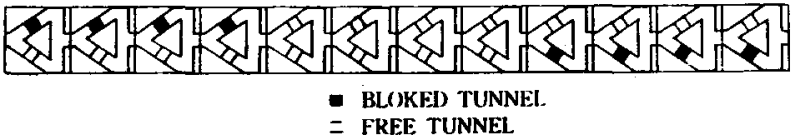

Fig. 1. Floor-plan of Maze I.

Each unit was placed on a $30 \mathrm{~cm} \times 27 \mathrm{~cm}$ plywood board and consisied of an isosceles triangle with a width of $4 \mathrm{~cm}$. Two apparently identical tunnels were put on both arms as shown in Fig. 1. Although it could not be detected from outside, one of the two tunnels was blocked so that the subject could not move a toy automobile through it.

Blocked tunnels were placed on the same side for the four entrance units and similarly for the four goal units. However, if blocks were on the right side of the goal units, for example, other four blocked tunnels were on the left side of the entrance units. Whether the block was on the right or left, was decided at random for each of the subjects. No blocks were placed for the free units.

A toy automobile of about $6 \mathrm{~cm} \times 3 \mathrm{~cm} \times 2 \mathrm{~cm}$ was used for the subject to trace the maze. The toy was especially used for the purpose that the subject would keep his interest relatively high during experimentation.

Procedure. The instruction given individually to the subject was, "I want you to trace the alley with this automobile from here (pointing to the starting-point) to the end of the alley (pointing to the goal-point). You have two alleys to choose at each of the choice point. On the first trial, however, I want you to go to whichever side you would like to. As you see, there is a tunnel on each of the two sides. The automobile can go through only one of them. If you fund a tunnel blocked, I want you to trace back to the choice point and go the other way. You will be given a bambi (a toy fawn made of straw) if you make no error throughout the trial." A bambi as reward was placed at the goal-point.

Training. The subject was given five trials a day and the alley chosen (i. e., right or left) at each choice point and the time spent for each trial were recorded.

Learning criterion. A learning criterion of three errorless trials in succession was used. However the training was discontinued if the criterion was not met in 21 trials. 


\section{RESULTS}

Median numbers of trials to learning criterion of each group was 10, 4, 4, 5.5, and 3.5 respectively in order of age. This decreasing trend in learning ability was significant at the five per cent level of confidence using the $H$-statistic $(H=11.6$, with $4 d f$.), although the main difference lay between the age-four group and the rest of the groups. Percentage of turns in each unit which agreed with the prescribed turns in the entrance units was computed for each subject. If the obtained score of a subject was greater than 51 per cent, the subject was called, 'entrance-directed' or 'entrance-oriented.' Percentages of 'entrance-directed' subjects were calculated per unit and were shown in Fig. 2 for different age groups.

In the entrance units, especially in unit 1 , there was observed a relatively large number of entrance-directed subjects in older groups as compared with young groups. On the other hand, younger subjects indicated a higher 'entrance-directedness' or, it may be called, 'entrance gradient' in free units than older subjects. A rapid decrease in the entrance gradient was shown from unit 9 to unit 10 in the goal path for all ages.

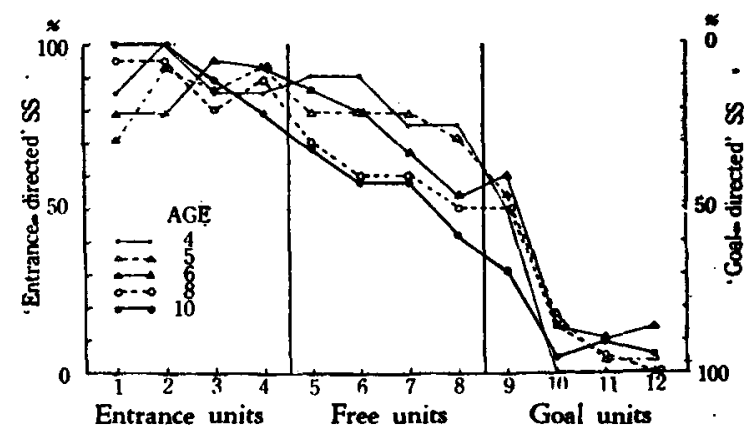

Fig. 2. Proportions of 'entrance-directed' subjects in each unit of Maze $I$ in pre-criterion trials. 'Entrance-directed' subjects are those who had more than $51 \%$ agreements with the entrance turn.

Table 1 indicates the number of subjects who had more than 51 mean per cent agreement with the entrance turn in free units for each of the age groups.

A slight decrease of the effect of the entrance path was found as a function of age but the overall trend was not significant by the use of chi-square (chi-square $=7.80$ with $4 d f$.). However, when the younger groups aging from four to six were combined against the rest of the groups, a significant chi-square was obtained (the statistic $=7.37$ with $1 d f$.).

Table 1

Proportions of 'Entrance-directed' and ' Goal-directed' Subjects for Each Age Group in Free Units of Maze I during Pre-criterion Trials

\begin{tabular}{cccccc}
\hline \multicolumn{1}{c}{ Age } & 4 & 5 & 6 & 8 & 10 \\
\hline Entrance-directed & 85 & 89 & 79 & 55 & $68 \%$ \\
Goal-directed & 15 & 11 & 21 & 45 & $32^{\%}$ \\
\hline$N$ & $20^{*}$ & $28^{*}$ & 28 & 40 & $19^{* *}$
\end{tabular}

$*$ In groups of ages four and five, two subjects failed to meet learning criterion.

** In group of age 10, there was a subject who made no errors at all.

These five subjects were excluded in computation.

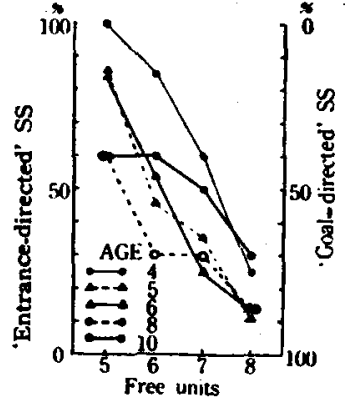

Fig. 3. Proportions of 'entrance-directed' subjects in free units of Maze I in three criterion trials.

The same analysis was made with regard to the last three trials for those who completed learning criterion. The number of subjects whose turning responses in free units 
agreed with the prescribed turn of the entrance path more than twice (i.e., more than 51 per cent) are shown in Fig. 3 for each unit as a function of age. The figure indicates a rapid diminution in entrance gradient from unit 5 to unit 8 as compared with that prior to learning criterion for each of the groups. Here again the effect of the entrance turn decreased with age except that the oldest age group showed a relative increase in entrance gradient.

Percentages of subjects whose free unit responses agreed more than 51 per cent (i.e., more than twice out of three times) with correct entrance turn in the last three criterion trials were computed against age as indicated by Table 2 . It was shown that

\section{Table 2}

Proportion of 'Entrance-directed' and 'Goal-directed' Subjects for each Age Group in Free Units in Maze II during Criterion Period.

\begin{tabular}{cccccc}
\hline Age & 4 & 5 & 6 & 8 & 10 \\
\hline Entrance-directed & 70 & 36 & 36 & 45 & $47 \%$ \\
Goal-directed & 30 & 64 & 64 & 75 & $53^{\%}$ \\
\hline $\mathrm{N}$ & 20 & 28 & 18 & 20 & 19 \\
\hline
\end{tabular}

the effect of the entrance path on free unit turns decreased with age eight and then increased at age 10. The overall differences in proportion among the age groups were significant at the five per cent confidence level by the use of chi-square (chi-square $=9.99$ with $4 d f$.).

The effect of the entrance path on free unit turns decreased

from the pre-criterion trials to the three criterion trials. This decrease was tested for significance using McNemar's chi-square for correlated proportions disregarding the unit differences (3). The obtained statistic was 1.29 for age four; 15.00 for age five; 10.29 for age six; 6.00 for age eight; and 4.00 for age 10 with one $d f$. for each of the chisquares. All the differences except that for the youngest group were significant at the five per cent level.

\section{EXPERIMET II}

In Experiment I, the maze was visually shown and the subject moved the body along the apparatus in tracing the alley. On the other hand, no visual cues were available and the body movement was restricted in Experiment II. For this purpose a finger maze was used. The blind-fold subject was seated in front of the table where the finger maze was placed. This maze called Maze II had the same structure as Maze I but the size was so small that the subject could easily stretch his arm from the starting-point to the goalpoint without moving the body. The alleys were made of vinyl strings being placed on a $27.5 \mathrm{~cm} \times 46 \mathrm{~cm}$ plywood board as shown in Fig. 4. Thumb-tacks were used as blocks for cul-de-sac. The same reward as in the previous experiment was utilized: The subjects used are indicated below :

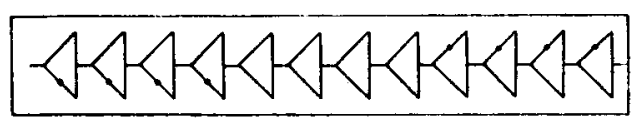

Fig. 4. Floor-plan of Maze II. Dots indicate blocks.

Maze II was presented before the blind-fold subject. The same instruction, the training procedures and the same criterion of learning were given as in Experiment I except that the subject in this experiment was instructed to trace the maze with his finger along the alley. In addition, the subject was given preliminary training using a three-unit unilinear maze of the same pattern as Maze II. After completion of learning criterion, the subject had to tell the experimenter how he succeeded in maze learning. 


\section{RELULTS}

Median numbers of trials to reach learning criterion was 18 for age five; 17 for age six; 9 for age eight; 7 for age 10 and 3 for adults. Again a decrease in trial numbers was observed as in Maze $I$. However, the trend was more distinct. A chisquare of 42.37 with four $d f$. using the H-test was obtained for testing the trend, indicating a statistical significance at the one per cent level.

Proportions of subjects who failed to meet learning criterion were greater in the finger maze than in the visual maze. There were eight such subjects out of 20 (i.e. 40 $\%$ ) for age five, 10 out of 25 (i.e. 40\%) for age six for Maze II, while there were only two subjects for each of groups of ages four and five (i.e. $9 \%$ and $7 \%$ respectively) for Maze I.

Proportion of agreement with the prescribed entrance turn was computed in each unit for each of the subjects. Proportions of those subjects who had more than 51 per cent agreements were then plotted against each unit for different age groups as shown in Fig. 5. In the entrance units the proportion was greater in younger groups (nearly 100 per cent) than in older groups. A great diminution in entrance gradient appeared from units 9 through 10 in the goal path for all the subjects. In the free units there was an observable tendency that younger subjects showed a greater entrance gradient than older subjects.

Table 3 indicates proportions of subjects in each of the groups whose average percentages of agreements with the entrance turn was more than 51 per cent in the free path. The obtained decrease in entrance gradient as a function of age was significant at the five per cent level by the use of chi-square (the statistic $=9.74$ with 4 df.). The same pro-

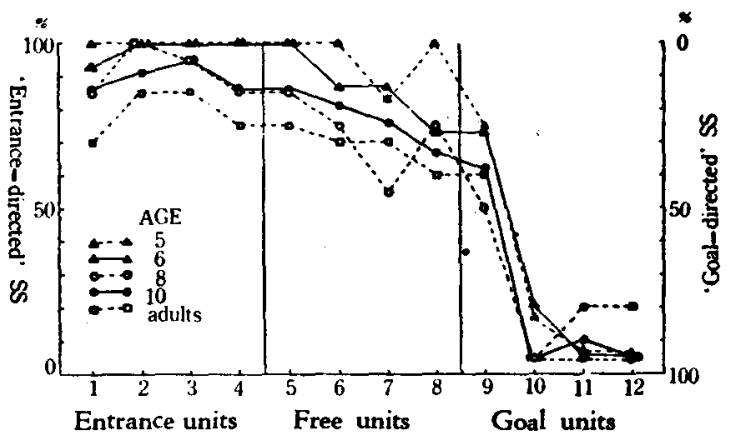

Fig. 5. Proportions of 'entrance-directed' subjects in each unit of Maze II in pre-criterion trials.

Table 3

Proportions of 'Entrance-directed' and ' Goal-directed ' Subjects for Each Age Group in Free Units of Maze II during Precriterion period.

\begin{tabular}{crrrrr}
\hline Age & 5 & 6 & 8 & 10 & Adult \\
\hline Entrance-directed & 100 & 100 & 70 & 81 & $70 \%$ \\
Goal-directed & 0 & 0 & 30 & 19 & 30 \\
\hline $\mathrm{N}$ & $12^{*}$ & $15^{* *}$ & 20 & 21 & 20 \\
\hline
\end{tabular}

* does not include eight subjects who failed to meet criterion. ** does not included 10 such subjects.

Table 4

Proportions of 'Entranc-directed' and 'Goal-directed' Subjects for Each Age Group in Free Units of Maze II during Criterion Period.

\begin{tabular}{cccccc}
\hline \multicolumn{1}{c}{ Age } & 5 & 6 & 8 & 10 & Adult \\
\hline Entrance-directed & 83 & 60 & 45 & 33 & 40 \\
Goal-direction & 17 & 40 & 55 & 67 & $60 \%$ \\
\hline N & 12 & 15 & 20 & 21 & 20 \\
\hline
\end{tabular}
portion was also computed for the last three criterion trials and is shown in Table 4. 
Again a decrease in entrance gradient was obtained but the chi-square just failed to reach the sinificance level $(\mathrm{p}=.06)$.

A clear diminution in entrance gradient, i.e. entrance-directed responses, from the pre-criterion period to the criterion period was shown for all the groups. Statistically significant differences were obtained for the groups except for two groups of ages five and eight. McNemar's chi-square (3) used was 2.00 for age five; 6.00 for age six; 2.27 for age eight; 10.00 for age 10 and 6.00 for adults.

Table 5 shows the classification of introspection concerning learning clues, given

Table 5

Learning Clues in Maze II Based on Introspection for Each Age Group.

\begin{tabular}{cccccc}
\hline Age & 5 & 6 & 8 & 10 & Adult \\
$\begin{array}{c}\text { Numerical clues } \\
\begin{array}{c}\text { Ambiguous clews } \\
\text { or feelings }\end{array}\end{array}$ & 0 & 13 & 40 & 48 & 70 \\
No answers & 100 & 7 & 15 & 4 & 0 \\
\hline N & 12 & 15 & 20 & 21 & 20 \\
\hline
\end{tabular}
by the subjects who met learn. ing criterion. By 'numerical clues' was meant those subjects who found that a certain num. ber of the same turns should precede before they had to switch their turns to the reverse direction on their way to the goal. Those who reported that they succeeded in learning the maze by shifting their turns at

a certain point who could not mention where it was with certainty, were categorized as 'ambiguous clues or feelings'. As a matter of fact, turning points which these subjects reported without confidence disagreed most of the times with the actual turning points made in the last criterion trials. Those who could not mention any learning clues were called 'no answers'. The percentage of the subjects who reported to have solved the maze by 'numerical clues' increased with age, while those who did not mention any clues, reduced in proportion very rapidly with age. The overall trend was statistically significant at the one per cent level of confidence (chi-square $=$ 71.72 with $8 d f$.).

In comparing the two maze learnings, median numbers of trials to reach learning criterion were used with age level constant. The non-parametric $U$ tests were utilized for testing the significance of the differences. The obtained critical ratio was 4.72 for age five; 5.59 for age six; 2.39 for age eight; and 4.31 for age 10, all the differences being significant beyond the five per cent level of confidence. In all the four pairs of groups, subjects on an average learned Maze I faster than Maze II. The other two groups, i.e. the group of age four in Experiment $I$ and the adult group in Experiment II could not be used for this comparison because they did not have comparative groups.

Times required to reach learning criterion were not analysed because of great individual variations.

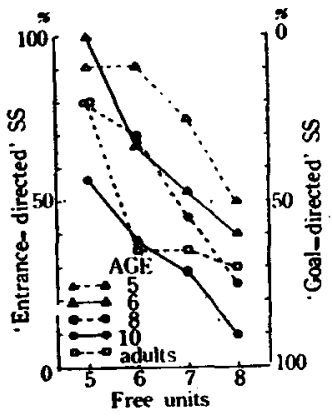

Fig. 6. Proportions of 'entrance-directed' sub. jects in free units of Maze II in three criterion trials.

\section{DISCUSSION}

Two different mazes I, and II of Haslerud pattern were used. Median trial numbers needed prior to learning criterion was found greater for Maze II without visual cues than 
for Maze I with visual cues available. Thus visual cues were found to be of importance in maze learning. In either of the mazes, younger subjects took more trials to reach learning criterion than older groups. This finding, although nothing new, was in agreement with McGinnis' (2).

The effect of the prescribed turn in the entrance path on turning responses in the middle free units was shown to decrease with age in pre-criterion learning. This would be due to the fact that younger subjects would have persisted in free units in turning to the reinforced side of the entrance path, while older subjects would anticipate the goal turn and would adjust their turns to that direction.

Although a similar relationship was found in free units during the three criterion trials in Experiment $I$, the oldest group of age 10 showed a relatively high entrance gradient as compared with the next young subjects. Thus we may classify all the groups into three categories; (1) those who persisted in 'entrance-directed' turn even in criterion trials, (group of age four), (2) those whose 'entrance-directed' responses decreased rapidly from pre-criterion trials to criterion trials (group of age five to six and eight), and (3) those whose 'entrance-directed' responses did not show any significant decrease in criterion trials (groups of age 10). It was believed that for those who belonged to category (2) a cognitive structure of the maze increased its clearness with trials to the effect that no errors would be made if they shifted their turns to the reverse direction in the middle part of the maze. In later trials, however, they found that the shift should be made closer to the goal than to the entrance. Thus, it is believed, the shift of turn approached the goal in the last three criterion trials in comparison with the previous trials. In other words, 'goal-directed' responses appeared later in the maze during the criterion period than in the pre-criterion period. This would explain why a relatively high degree of goal gradient was observed for the groups of ages five and six, and age eight, as compared with the younger group of age four. The latter group which belongs to category (1) above, on the other hand, could not perhaps have as articulated a cognition of the maze as the older groups. They persisted in 'entrance-directed' responses and in consequence they made an error in unit 9. However, after a few repetitions of errors in unit 9, 'goal-directed' responses appeared in units 7 and 8 in free units or responses either goaldirected or entrance-directed were made after much hesitation in the same units and finally most of the subjects succeeded in making correct, i.e. goal-directed, turns in unit 9 and the rest of the units. This kind of behavior was observed quite often. It was assumed that the subject's shift of turn was made on his rough idea of distance either from goal or entrance. The subject's vague cognition of the maze and his persistence of the originally rewarded turns, i.e. the 'entrance-directed' turns, would explain why an entrance gradient rather than a goal-gradient was shown in tree units for this group.

The group of age 10 who belonged to category (3), on the other hand, would have an articulated knowledge of the maze structure fairly early in trials in comparison with the other groups and they would have found that they would be correct whether they responded to the right side or to the left, in the free units. This would explain why in average their turning responses were shifted from 'entrance-directed' to 'goal-directed' in about the middle of the free units, and no significant increase in goal gradient was observed in the criterion trials as compared with the pre-criterion trials.

A very similar relationship was found between the three response categories and age in Maze II, although the chi-square just failed to reach the five per cent significance 
level. Three groups of ages five, six and eight belonged to category (1), and the age 10 group to category (2) and the adult group to category (3). As Maze II was harder than Maze I, the response pattern obtained from the Maze II subjects was retarded to a lower level. For example, the adult group in this experiment showed similar responses to those of the age 10 group in the previous experiment.

Moreover, it should be mentioned that a considerable number of subjects of ages five and six could not reach learning criterion in Experiment II. This seems to indicate that a new response category should be added beside the three categories already mentioned. Those who belonged to this group, i.e. those who failed to meet learning criterion, persisted in 'entrance-directed' responses up to unit 9. As an entrance-directed response was incorrect and it was the first error, the incorrect response in unit 9 now began to be a sign of shifting thereafter to 'goal-directed' responses. Their knowledge of the maze was simply to persist in the entrance-directed response up to the point where the response was blocked and then to shift their turns until goal. They could not learn where that critical turning point was in order to reach the goal without errors. They could not anticipate the correct turns in the goal units.

Thus four developmental patterns or categories were observed with respect to the free units in the maze learning; ( 0 ) those who could grasp the maze structure only after an error was made in the first unit of the goal units, (1) those whose performance showed a relatively high degree of entrance gradient, (2) those whose responses were affected by the goal responses especially during the criterion period as compared with younger and even with older groups, (3) those responses did not show any particular kind of gradient.

The present results as a whole were not in accordance with those of Haslerud in which the free units had almost complete agreements with the goal turn. His subjects' performance seemed to be higher in the response category. However, the direct comparison may be erroneous because the two situations were not entirely the same.

\section{SUMMARY AND CONCLUSIONS}

Two types of a 12-unit unilinear maze of the Haslerud pattern were used for a developmental study of entrance and goal gradients in human maze learning. A half of the group was required to learn a maze pattern in which the first four units were blocked at the right alley, the following four units were not blocked at all and the last four were blocked at the left alley. The other half had to learn the reverse, i.e. the entrance units to right, the goal units to left and the middle units free.

Four groups of different ages from four through 10 learned Maze $I$ and five groups of ages from five to adult were tried on Maze II were, contrary to Maze I, no visual cues were available. The following results were obtained : 1) The number of trials to learning criterion was greater for the Maze II groups than for the Maze I groups. 2) In both mazes, the number of trials to criterion decreased with age. 3) The entrance gradient was dominant over the goal gradient in free units for all groups especially for younger groups. 4) In the three criterion trials, the entrance gradient was still high in younger groups in comparison with older groups. 5) During the same period the shift from 'entrance-directed' responses to 'goaldirected' responses appeared in the free units for most of the groups. 6) Learning clues were more conceptual in older groups as compared with younger groups.

\section{REFERENCES}

1. Haslerud, G. M. Form and interaction of goal and entrance gradients in a unilinear maze. J. Psychol., $1945,20,249-257$.

2. McGinnis, E. The aquisition and interference of motor-habits in young children. Genet. Psychol. Monogr., 1929, 6, 209-311.

3. McNemat, Q. Psychological statistics. New Yock: John Wiley \& Sons, 1949.

MS. received III 14,57 . 